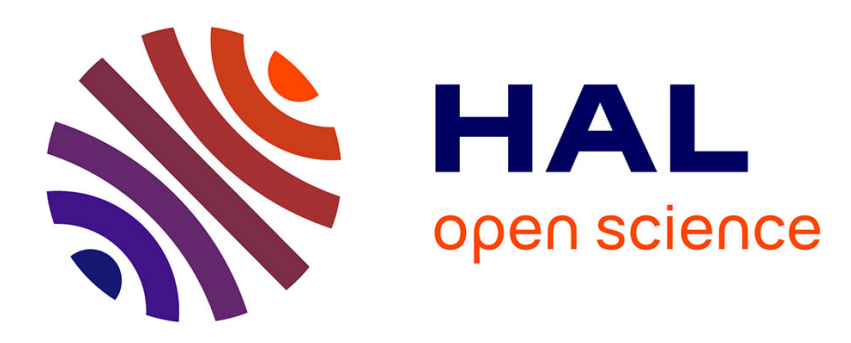

\title{
Lattice anisotropy, electronic and chemical structures of uranyl carbonate, UO2CO3, from first principles
}

\author{
Samir F. Matar
}

\section{To cite this version:}

Samir F. Matar. Lattice anisotropy, electronic and chemical structures of uranyl carbonate, UO2CO3, from first principles. Chemical Physics, 2010, 372 (1-3), pp.46-50. 10.1016/j.chemphys.2010.04.020 . hal-00490995

\section{HAL Id: hal-00490995 \\ https://hal.science/hal-00490995}

Submitted on 10 Jun 2010

HAL is a multi-disciplinary open access archive for the deposit and dissemination of scientific research documents, whether they are published or not. The documents may come from teaching and research institutions in France or abroad, or from public or private research centers.
L'archive ouverte pluridisciplinaire HAL, est destinée au dépôt et à la diffusion de documents scientifiques de niveau recherche, publiés ou non, émanant des établissements d'enseignement et de recherche français ou étrangers, des laboratoires publics ou privés. 


\title{
Lattice anisotropy, electronic and chemical structures of uranyl carbonate, $\mathrm{UO}_{2} \mathrm{CO}_{3}$, from first principles
}

\author{
S. F. Matar* \\ CNRS, Université de Bordeaux, ICMCB, 87 avenue du Docteur Albert Schweitzer, \\ F-33608 Pessac Cedex, France
}

This work is dedicated to the memory of Fouad E. Matar ${ }^{\dagger 2005}$.

\begin{abstract}
Band theoretical results are presented on $\mathrm{UO}_{2} \mathrm{CO}_{3}$, based on computations within the density functional theory. The equation of state is obtained with equilibrium lattice properties in agreement with experiment. For isotropic volume change the bulk modulus amounts to $176 \mathrm{GPa}$. A higher value for anisotropic compression along the linear uranyl characterizes its incompressibility. The electronic band structure shows a semiconducting behavior $(\sim 2 \mathrm{eV}$ band gap) with little band dispersion. From chemical bonding plots and electron localization function mapping, oxygen atoms are found to preferentially bind with uranium for one sublattice and to carbon forming the carbonate ions for the 2 others. This further illustrates the ionic-like $\left(\mathrm{UO}_{2}\right)^{2+}\left(\mathrm{CO}_{3}\right)^{2-}$ chemical picture.
\end{abstract}

Key words: Uranyl carbonate, Rutherfordine, DFT, VASP, ASW, ELF, COOP

\section{Introduction}

Binary and ternary uranium natural and synthetic compounds are known and characterized (mainly X-ray diffraction) since the first half of last century $[1,2,3]$. For such technology relevant systems, establishing the electron band structure, the equation of state (EOS) as well as the properties of chemical bonding are useful both at the fundamental and application levels like for better handling and disposal of chemical residues occurring as secondary products in nuclear reactors. This can be accurately carried out using quantum

* E-mail: matar@icmcb-bordeaux.cnrs.fr; fax: +33 540002761. 
mechanical computation methods built within the density functional theory DFT $[6,7]$. Such investigations have been undertaken for well known tetravalent uranium oxide, $\mathrm{UO}_{2}[8,4]$ and hexavalent uranium based fluorides, $\mathrm{UF}_{6}$ [5], oxides, $\mathrm{CaUO}_{4}[9]$ and $\mathrm{CdUO}_{4}[10]$ as well as oxyfluorides $\mathrm{UO}_{2} \mathrm{~F}_{2}$ [11] in which the role of uranyl cation was highlighted. Theoretical works on uranyl peroxides are also reported [12].

Uranyl carbonate $\mathrm{UO}_{2} \mathrm{CO}_{3}$, also known as Rutherfordine mineral, was characterized by X-ray diffraction by Christ et al. [2]. It is orthorhombic with Pmmn space group and two formula units (fu) per cell. Fig. 1 shows the structure with the coordination polyhedra of $\mathrm{U}$ and $\mathrm{C}$. While the former is found in an octa-coordinated oxygen polyhedron, carbon is in a planar oxygenated environment. Along the b-axis, this forms aligned uranyl $\left(\mathrm{UO}_{2}\right)$ group and orthogonal triangular $\left(\mathrm{CO}_{3}\right)$ carbonate. From the experimental lattice parameters in Table 1 (left hand column [2]), oxygen is dispatched into 3 sites labeled O1, O2 and O3. The nearest neighbor distances point to O1 and O3 in the neighborhood of carbon forming $\left(\mathrm{CO}_{3}\right)$; nevertheless they contribute to the hexagonal planar part of uranium 8-fold coordination polyhedron with the 2 apical O2 forming the uranyl unit as it is highlighted in the sketch. Hence the structure should show anisotropic features. The purpose of this paper is to present results within DFT on the equation of state EOS for isotropic and anisotropic volume changes and to discuss issues in the electronic band structure with corresponding atom resolved density of states and chemical bonding properties.

\section{Computational framework}

Within DFT, calculations of the EOS and electron localization were carried out using a pseudo-potential (PP) approach with the Vienna ab initio simulation package (VASP) code [13]. Projector augmented wave (PAW) [14,15] potentials built within the local density approximation (LDA) [16] as well as the generalized gradient approximation (GGA) [17] were used. Besides of being more accurate than ultra-soft PP's, PAW potentials treat the f orbitals as valence states. The optimization of the structural parameters is performed until the forces on the atoms are less than $0.02 \mathrm{eV} / \AA$ and all stress components are below $0.003 \mathrm{eV} / \AA^{3}$. Calculations are converged at an energy cut-off of $500 \mathrm{eV}$ for the plane-wave basis set. Brillouin zone (BZ) integrals are approximated [18] using k-point grids with a starting mesh of $4 \times 4 \times 4$ up to $8 \times 8 \times 8$ for best convergence and relaxation to zero strains. Further, from the calculations a mapping of the electrons in the lattice can be obtained using the electron localization function (ELF) scheme, introduced by Becke and Edgecombe [19]. It allows determining the amount of localization of electrons with respect to the free electron gas distribution. The dimensionless ELF magnitude ranges 
from 0 to 1 with $\mathrm{ELF}=\frac{1}{2}$ corresponding to the free electron gas distribution. These three extreme situations will be illustrated by three sets of colors : ELF $=0$ with no electron localization are shown with blue contours, ELF $=1$ with strong localization have red contours and ELF $=\frac{1}{2}$ for the free electron gas with green contours.

All-electron calculations, necessary to explore the whole valence band (VB) / conduction band (CB) spectrum as well as a detailed study of the chemical bonding, were performed using the scalar-relativistic implementation of the augmented spherical wave (ASW) method [20,21]. As it was established from the PP calculations that the GGA provided the correct ground state (cf. next section), the computations used GGA scheme [17]. In the ASW method, the wave function is expanded in atom-centered augmented spherical waves, which are Hankel functions and numerical solutions of Schrödinger's equation, respectively, outside and inside the so-called augmentation spheres. In the minimal ASW basis set, we chose the outermost shells to represent the valence states and the matrix elements were constructed using partial waves up to $l_{\text {max }}+1=4$ for $\mathrm{U}$ including $5 \mathrm{f}$ sub-shell and $l_{\max }+1=2$ for $\mathrm{C}$ and O. The ASW method uses the atomic sphere approximation (ASA) which assumes overlapping spheres centered on the atomic sites whose volume is equal to the cell volume. In order to represent the correct shape of the crystal potential in the large voids of the crystal structure (Fig. 1), additional augmentation spheres were inserted to avoid an otherwise too large overlap between the actual atomic spheres. These are called empty spheres (ES) described as pseudo-atoms with with zero atomic number and basis set $l_{\max }$. $1=2$ up to 3 , allowing them to receive charges from the neighboring atomic species. The choice of these sites as well as the augmentation radii are automatically determined using the sphere-geometry optimization algorithm [24]. All calculations are started for neutral atom configurations. Convergence is obtained when negligible variations for the charges $\left(\Delta \mathrm{Q}=10^{-8}\right)$ and for the total energy $\left(\Delta \mathrm{E}=10^{-7} \mathrm{eV}\right)$, are observed between two successive iterative cycles. Self-consistency was achieved by a highly efficient algorithm for convergence acceleration [25]. The BZ integrations were performed using the linear tetrahedron method with up to $280 \mathrm{k}$-points generated from 2744 points in the irreducible wedge $[14,21]$. Information about the nature of the chemical bond between two atomic constituents $(i, j)$ is qualitatively disussed from the overlap population analysis: $\mathrm{S}_{i j}$ leading to the crystal orbital overlap population (COOP) [26] criterion. In the plots positive, negative and zero COOP magnitudes indicate bonding, anti-bonding and non-bonding interactions respectively. 


\section{Pseudo-potential calculations}

\subsection{Geometry optimization}

Starting from the experimental data in Table 1, a full geometry relaxation was carried out with PAW potentials constructed both with LDA and GGA exchange-correlation functionals for a search of the correct structure in comparison with experiment. At energy convergence and minimum lattice strains, GGA-PAW calculations led to better agreement of the cell volume than LDAPAW ones which give far smaller values; this can be assigned to the overbinding behavior of the LDA. Then the calculational results in this paper are based on the GGA gradient exchange-correlation functional. From the right hand side column of Table 1 giving the relaxed lattice parameters, there is some deviation versus experimental data on the mineral. The most significant trend is the reduction of the $\mathrm{b}$ axis magnitude caused by smaller $\mathrm{U}-\mathrm{O} 2$ spacing: $\Delta \mathrm{d}=$ $0.14 \AA$. However we note that the calculated value of $1.79 \AA$ remains within range of $\mathrm{U}-\mathrm{O}$ distance in the synthetic material [27] as well as in other uranyl based compouds [28]. On the contrary, along a and c axes less changes are observed except for a slight distortion of the $\mathrm{CO}_{3}$ unit as it can be inferred from the C-O1 distance change from 1.28 to $1.29 \AA$. The $\mathrm{U}$ - C distance increases so that the corresponding oxygenated units tend to separate, indicating a trend to a more ionic system.

\subsection{Mapping of electron localization}

An illustration of the results is obtained from the electron localization function, ELF. Fig. 2 shows the ELF maps for a vertical plane along the b-axis as in Fig. 1 and across $\mathrm{CO}_{3}$. In Fig. 2a the linear "O2-U-O2" entities are observed with strong localization around them (red contours) and no localization in between. The trace of the triangular shaped carbonate groups can be equally observed but $\mathrm{CO}_{3}$ assemblies are better seen in Fig. $2 \mathrm{~b}$ for a plane orthogonal to the b-axis, showing 4 adjecent cells in the projection for sake of clarity. No localization around $\mathrm{C}$ is found while the two oxygen sites, O1 and O3, have the largest localization. Contrary to Fig. 2a, the feature of green contours and little presence of blue spots in Fig. 2b is noteworthy. This means that there is mainly a free electron like behavior along the plane, between the $\mathrm{CO}_{3}$ groups. Beside showing separate $\left(\mathrm{UO}_{2}\right)$ and $\left(\mathrm{CO}_{3}\right)$ complex species, the blue spots between them feature the ionic and insulating character of the chemical system. Although of hexavalent character as it will be shown from the band structure section below, red spots are seen around uranium. This is an artefact from the construction of the PAW-potentials accounting for $5 \mathrm{f} \mathrm{U}$ 
states.

\section{3 $E(V)$ equation of state}

It needs to be mentioned that the calculated total energy pertains to the cohesive energy within the crystal because the solution of the Kohn-Sham DFT equations yields the energy with respect to infinitely separated electrons and nuclei. In as far as the zero of energy depends on the choice of the pseudopotentials, somehow it becomes arbitrary; i.e. it is shifted but not scaled. However the energy derivatives remain unaltered as well as the equations of state. For this reason one needs to establish the EOS and extract the fit parameters for an assessment of the equilibrium values. This is achieved from the energy versus volume $(\mathrm{E}, \mathrm{V})$ curves around the minima which can be fitted using Birch [30] equation of state (EOS) to the 3rd order:

$E(V)=E_{0}\left(V_{o}\right)+\frac{9}{8} V_{o} B_{o}\left[\left(\frac{V_{o}}{V}\right)^{\frac{2}{3}}-1\right]^{2}+\frac{9}{16} B_{o}\left(B^{\prime}-4\right) V_{o}\left[\left(\frac{V_{o}}{V}\right)^{\frac{2}{3}}-1\right]^{3}$.

$\mathrm{E}_{o}, \mathrm{~V}_{o}, \mathrm{~B}_{o}$ and $\mathrm{B}^{\prime}$ are the equilibrium energy, volume, bulk modulus and its pressure derivative, respectively. The Birch EOS is normally employed by assuming the following trend: the larger the value of $\mathrm{B}_{o}$, the harder is the material. Beside isotropic compression we hypothesize selective compression along the b-axis (cf. Fig. 1) with a volume change as in isotropic compression. We note that this procedure, applied for high structural anisotropy systems such as hexagonal graphite and BN, is experimentally used in establishing the EOS of less anisotropic systems such as $\mathrm{Al}_{4} \mathrm{C}_{3}$ [31]. The resulting energy versus volume curves and the fit results are shown in Fig. 3. The curves have a quadratic behavior with a less 'open' curve for anisotropic compression, due to the larger energy variation with volume, i.e. there is larger energy cost for the same volume change. The fit results show that the isotropic compression gives a closer equilibrium volume to experiment than relaxed parameters meaning that the ground state is better reproduced with the EOS. The bulk modulus magnitude, $\mathrm{B}_{o}^{i s o}=176 \mathrm{GPa}$, comes close to that of uranium dioxide $\mathrm{UO}_{2}[4]$ and similar isotropically compressed systems $[9,11]$. Looking at the anisotropic compression along $\mathrm{UO}_{2}$ (b-axis), the fit values show a smaller volume and less stable system but the relevant result is the enhancement of the bulk modulus with a value of $540 \mathrm{GPa}$, close to that calculated in the oxyfluoride [11]. This indicates the incompressibility of the uranyl unit. Note that for a compression within the plane a bulk module of $\sim 300 \mathrm{GPa}$ is obtained, meaning that the longer U-O1, U-O3 distances allow for a higher compressibility. The carbonate ion is found to play a secondary role in this respect. The reason why the overall isotropic bulk modulus is small is due to the larger separation between these complex ions, note that from Table 1 the $\mathrm{U}-\mathrm{C}$ distance is close to $3 \AA$, whence 
the ease of their response to external constraints. It can be mentioned that these complex ions $\left(\mathrm{UO}_{2}^{++}, \mathrm{CO}_{3}^{--}\right)$, are formed of atomic species which bind chemically, i.e., they constitute micro-chemical systems with size and shape but they remain embedded in an array of an extended crystal system. So one can invoke atom - cluster - resolved incompressibility. Lastly we point out that for the pressure derivatives of the bulk modulus $\mathrm{B}^{\prime}{ }_{\text {iso }}=4$ and $\mathrm{B}_{\text {aniso }} \sim$ 9. Whereas the first value is the magnitude usually obtained, the anisotropic B' indicates an enhanced stiffness of the system [32].

\section{All-electron calculations}

\subsection{Band structure and density of states}

At self consistency, a large charge transfer of $\sim 3.5$ electrons is observed from uranium towards the other species as well as to the ES. This expected trend is not exhibiting an ionic character such as $\mathrm{U}^{6+}$, rarely observed in the framework of the calculations. However this will be indirectly seen from the electronic band structure plotted along the main directions of the irreducible wedge of the BZ in Fig. 4. Along the vertical y-axis, the energy is counted with respect to the top of the valence band $\left(\mathrm{E}_{V}\right)$ because the system is insulating with $\sim 2$ $\mathrm{eV}$ band gap which is direct between $\Gamma_{V B}$ and $\Gamma_{C B}$. In fact such an assignemnt is made difficult due to the little dispersive character of the bands. In order to better discuss them the site projected density of states DOS are shown to the left of the bands. The low energy lying bands can be assigned to oxygen which behave differently for $\mathrm{O} 2$ versus $\mathrm{O} 1, \mathrm{O} 3$ whereby the latter are at much lower energy than the former. Then O1 and O3 behave as "more electronegative" than O2. Towards the top of the VB the O2 and uranium PDOS have similar shapes, followed at lower energy by the $\mathrm{C}$ and O1,O3 PDOS. This should be made explicit from the chemical bonding analysis below.

\subsubsection{Effect of enhanced correlation}

The very little dispersion of the bands underlines high localization, which can also be concluded from the narrow shape of the PDOS peaks. Then the use of an electron-gas based functional in present calculations might constitute

a drawback. For a check we have carried out an additional calculation by introducing the Hubbard repulsive parameter U. With values used by Freyss et al. for the study of $\mathrm{UO}_{2}$ and its defect structure, $\mathrm{U}=4.5 \mathrm{eV}$ and $\mathrm{J}=$ $0.5 \mathrm{eV}$ [33], the resulting band structure exhibits the same general features as in Fig. 4 with the difference of the band gap increase by $\sim 1 \mathrm{eV}$, i.e. leading to a more insulating system. The localization of the uranium $f$ bands 
in the CB is also the same. This probably arises from the fact that the system is involved with empty $f$ subshell of hexavalent uranium $\left(\mathrm{U}^{0}\right.$ : $[\mathrm{Rn}] 5 \mathrm{f}^{3} 5 \mathrm{~d}^{1} 7 \mathrm{~s}^{2}$ $\left.\rightarrow \mathrm{U}^{6+}:[\mathrm{Rn}] 5 \mathrm{f}^{0} 5 \mathrm{~d}^{0} 7 \mathrm{~s}^{0}\right)$. Therefore, the theoretical treatment using a scalarrelativistic calculational scheme and GGA functional used here should stand correctly.

\subsection{Chemical bonding}

The band structure results can be further illustrated by the chemical bonding properties using the COOP criterion [26]. As a general trend, the plots accounting for atom multiplicities in the structure, i.e. $2 \mathrm{U}, 2 \mathrm{C}, 2 \mathrm{O} 1,4 \mathrm{O} 2$ and 4 O3, are shown in Fig. 5 together with the corresponding partial DOS for $\mathrm{U}$ with $\mathrm{O} 1, \mathrm{O} 2$ and $\mathrm{O} 3$ and $\mathrm{C}$ with $\mathrm{O} 1, \mathrm{O} 2$ and $\mathrm{O} 3$. In both COOP plots, there is very little bonding below $-10 \mathrm{eV}$, i.e. in the energy ange of oxygen $s$ like states. A bonding character is observed all over the VB.This is indicative of the stability of the chemical system thanks to both its component entities: $\mathrm{UO}_{2}$ and $\mathrm{CO}_{3}$. In the energy range above $-10 \mathrm{eV}$, the chemical bond is ensured by the mixing of itinerant $\mathrm{U}$ states with O2-p states (Figs. 5a,b) and C,O1,O3 - $p$ states in Fig. 5-c,d; antibonding COOP appear in the CB. Interestingly the gap for the C,O DOS plot is larger than in the U,O one, i.e. the empty uranium $\mathrm{f}$ states at the bottom of the $\mathrm{CB}$ are responsible for the reduction of the electronic band gap. The top of the VB is dominated by the U-O2 bonding with antibonding counterparts within the CB. Little bonding of uranium with the $\mathrm{O} 1$ and $\mathrm{O} 3$ can be observed due to their large separation (cf. Table 1). $\mathrm{C}$ bonding is mainly with $\mathrm{O} 1$ and $\mathrm{O} 3$ constituting the carbonate group. As a general feature, the $\mathrm{C}-\mathrm{O}$ bonding is found larger than $\mathrm{U}-\mathrm{O}$ one. This can be related with the short $\mathrm{C}-\mathrm{O}$ bond distances as well as to the $C=O$ double bond character. Further the bonding and antibonding COOP for $\mathrm{C}-\mathrm{O}$ interactions are observed below $-5 \mathrm{eV}$ and at $\sim 5 \mathrm{eV}$ respectively. This resembles the M.O. diagram found for $\mathrm{CO}_{3}^{--}$in textbooks. These results further stress the ELF maps observed in Fig. 2.

\section{Conclusion}

In this work ab initio results on the electronic structure of $\mathrm{UO}_{2} \mathrm{CO}_{3}$ have been presented based on DFT computations with pseudo-potential and all electron methods aiming at i) providing information on its EOS and electron localization mapping, and ii) detailing the study of the band structure with weighting the bands, the site projected density of states and the chemical bonding characteristics. It has been mainly shown that the system is a relatively soft material if isotropically compressed. Large bulk moduli for anisotropic com- 
pressions along the complex uranyl and carbonate constituting entities, imply their incompressibilities. The band structure is characterized by a gap of $\sim$ $2 \mathrm{eV}$ at $\Gamma$ point occurring between $\mathrm{VB}[\mathrm{U}(\mathrm{f})-\mathrm{O}(\mathrm{p})]$ band and $\mathrm{CB}[\mathrm{U}(\mathrm{f})]$. Oxygen sublattices are found to behave differently according to their selective binding with $\mathrm{U}(\mathrm{O} 2)$ versus $\mathrm{C}(\mathrm{O} 1, \mathrm{O} 3)$. This complements the picture of a complex uranyl cation met in molecular chemistry community [34]. Finally it is proposed that this case study is a good example of structure $\leftrightarrow$ property (mechanical, electronic, covalent versus ionic bonding, ... ) relationship in materials science.

\section{Acknowledgments}

Discussions with Emer. Prof. Gérard Demazeau are gratefully acknowledged. Computational facilities were provided by the University Bordeaux 1 on main frame computers of M.C.I.A. (Mésocentre de Calcul Intensif en Aquitaine), financed by the Council of Aquitaine Region and the French Ministry of Research and Technology.

\section{References}

[1] W.H. Zachariasen, Acta. Cryst. 1,1948, 265.

[2] C.L. Christ, J. R. Clark, H.T. Evans Jr, Science, 121, 1955, 472.

[3] W.H. Zachariasen, Acta. Cryst. 1, 1948, 281.

[4] H. Y. Geng, Y. Chen, Y. Kaneta, M. Kinoshita, Phys. Rev. B 75, 2007, 054111.

[5] R. A. Evarestov, A. I. Panin, A. V. Bandura, Russian J. General Chem., 78, 2008, 1823.

[6] P. Hohenberg, W. Kohn, Phys. Rev., 136, 1964, 864.

[7] W. Kohn and L.J. Sham, Phys. Rev., 140, 1965, 1133.

[8] L.E. Roy, T. Durakiewicz, R.L. Martin, J.E. Peralta, G.E. Scuseria, C.G. Olson, J.J. Joyce, E. Guziewicz, J. Comput. Chem. 29, 2008, 2288.

[9] S.F. Matar, G. Demazeau, J. Solid State Chemistry, 182, 2009, 2678.

[10] S.F. Matar, Chem. Phys. Lett., 476, 2009, 213.

[11] S.F. Matar, Solid State Sci., 11, 2009, 1380.

[12] S. Ostanin, P. Zeller, Phys. Rev. B, 75, 2007, 073101.

[13] G. Kresse, J. Furthmüller, Phys. Rev. B 54, 1996, 11169. 
[14] P. E. Blöchl, Phys. Rev. B 50, 1994, 17953.

[15] G. Kresse, J. Joubert, Phys. Rev. B 59, 1999, 1758.

[16] D.M. Ceperley, B.J. Alder, Phys Rev Lett 45, 1980, 566.

[17] J. Perdew, K. Burke, M. Ernzerhof, Phys. Rev. Lett. 77, 1996, 3865.

[18] H. J. Monkhorst, J. D. Pack, Phys. Rev. B 13 (1976) 5188.

[19] A.D. Becke, K.E. Edgecombe, J. Chem. Phys. 92, 1990, 5397.

[20] A. R. Williams, J. Kübler, C. D. Gelatt, Phys. Rev. B 19, 1979, 6094.

[21] V. Eyert, The Augmented Spherical Wave Method - A Comprehensive Treatment, Lect. Notes Phys. 719 (Springer, Berlin Heidelberg 2007).

[22] P. E. Blöchl, Phys. Rev. B, 50, 1994, 17953.

[23] S. H. Vosko, L. Wilk, and M. Nusair. Can. J. Phys. 58, 1980, 1200.

[24] V. Eyert, K. -H. Höck, Phys. Rev. B 57, 1998, 12727.

[25] V. Eyert, J. Comput. Phys. 124, 1996, 271.

[26] R. Hoffmann, Angew. Chem. Int. Ed. Engl. 26, 1987, 846.

[27] D. T. Cromer, P. E. Harper, Acta Cryst. 8, 1955, 847.

[28] W.H. Zachariasen, Acta Cryst. 7, 1954, 795.

[29] G. Bester, M. Fähnle, J. Phys: Condens. Matter 13, 2001, 11541.

[30] F. Birch, J. Geophys. Res. 83, 1978, 1257.

[31] V. L. Solozhenko, O. O. Kurakevych, Solid State Commun., 133, 2005, 385.

[32] S. Raju, E. Mohandas, V. S. Raghunathan, J. Phys. Chem. Solids, 58 , 1997, 1367.

[33] M. Freyss, J. Durinck, B. Dorado, Colloque 3M, Saclay, 2-3 June 1008, France (unpublished).

[34] N. Iché-Tarrat, N. Barros, C. J. Marsden, L. Maron, Chem. Eur. J. 14 (2008) 2093. 


\begin{tabular}{|l|l|l|}
\hline $\mathrm{UO}_{2} \mathrm{CO}_{3}$ Pmmn ORIG.2 & Experimental $[2]$ & Calculated (this work) \\
\hline a-latt. const. $(\AA)$ & 4.296 & 4.264 \\
b-latt. const. $(\AA)$ & 9.205 & 8.929 \\
c-latt. const. $(\AA)$ & 4.845 & 4.842 \\
Atoms & & \\
$\mathrm{U} \pm\left(\frac{1}{4}, \frac{3}{4}, u\right)$ & $\mathrm{u}=0.750$ & $\mathrm{u}=0.765$ \\
$\mathrm{C} \pm\left(\frac{1}{4}, \frac{1}{4}, u^{\prime}\right)$ & $\mathrm{u}=0.601$ & $\mathrm{u}^{\prime}=0.619$ \\
$\mathrm{O} 1 \pm\left(\frac{1}{4}, \frac{1}{4}, u^{\prime \prime}\right)$ & $\mathrm{u}^{\prime \prime}=0.303$ & $\mathrm{u}^{\prime \prime}=0.314$ \\
$\mathrm{O} 2 \pm\left(\frac{1}{4}, y, z \frac{1}{4}, \frac{1}{2}-y, z\right)$ & $\mathrm{y}=0.960 ; \mathrm{z}=0.750$ & $\mathrm{y}=0.954 ; \mathrm{z}=0.755$ \\
$\mathrm{O} 3 \pm\left(x, \frac{1}{4}, z^{\prime} \frac{1}{2}-x, \frac{1}{4}, z^{\prime}\right)$ & $\mathrm{x}=0.021 ; \mathrm{z}=0.750$ & $\mathrm{x}=0.012 ; \mathrm{z}=0.750$ \\
Distances $(\AA)$ & & \\
$\mathrm{U}-\mathrm{O} 1 / \mathrm{U}-\mathrm{O} 2 / \mathrm{U}-\mathrm{O} 3$ & $2.43 / 1.93 / 2.52$ & $2.44 / 1.79 / 2.53$ \\
C-O1/C-O2/C-O3 & $1.28 / 2.43 / 1.28$ & $1.29 / 2.44 / 1.28$ \\
$\mathrm{U}-\mathrm{C}$ & 2.85 & 2.91 \\
Volume $\left(\AA^{3}\right)$ & 191.59 & 184.36 \\
$\mathrm{~B}_{o}^{\text {iso. }(\mathrm{GPa})}$ & & 176 \\
$\mathrm{~B}_{o}^{\text {aniso.-b }(\mathrm{GPa})}$ & & 540 \\
\hline
\end{tabular}

Table 1

Lattice parameters of $\mathrm{UO}_{2} \mathrm{CO}_{3}$ 


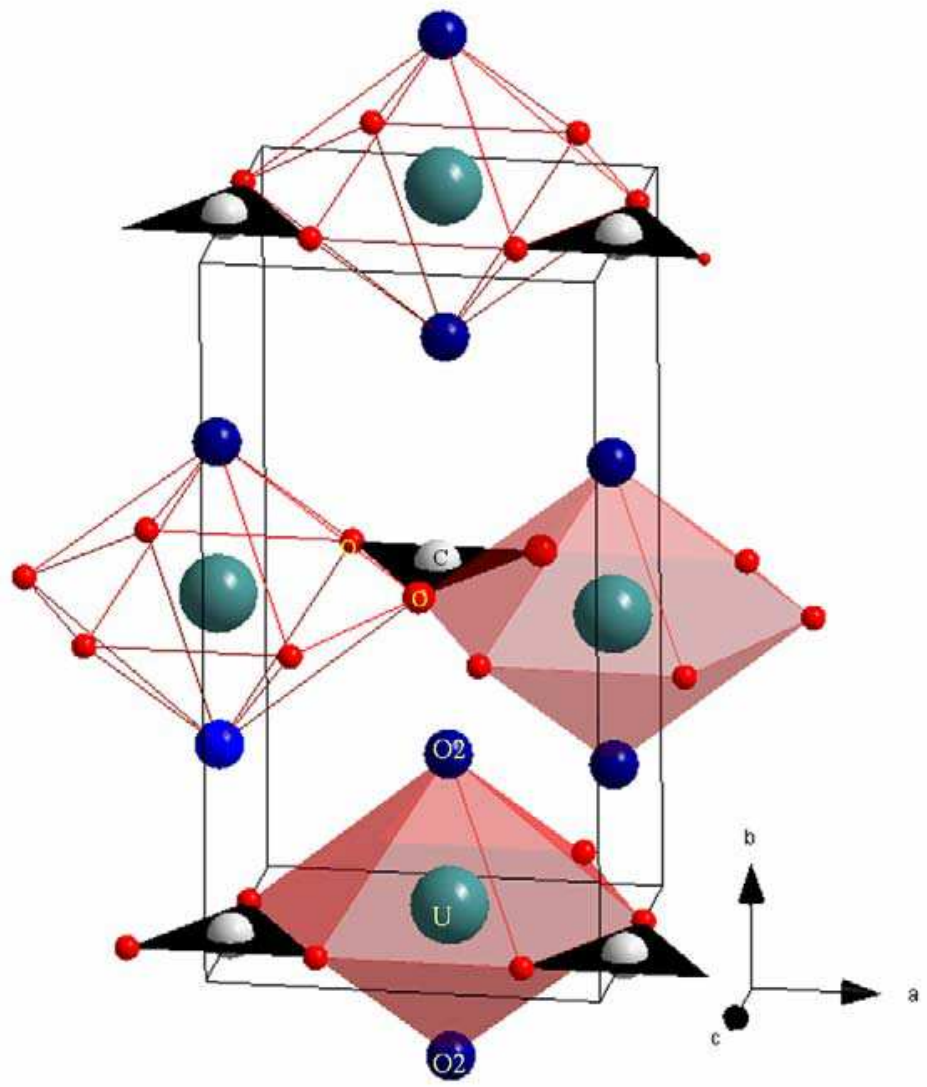

Figure 1. Structure of $\mathrm{UO}_{2} \mathrm{CO}_{3}$ showing the U-O and C-O coordination polyhedra and highlighting the linear $\mathrm{O} 2-\mathrm{U}-\mathrm{O} 2$ uranyl ion. 


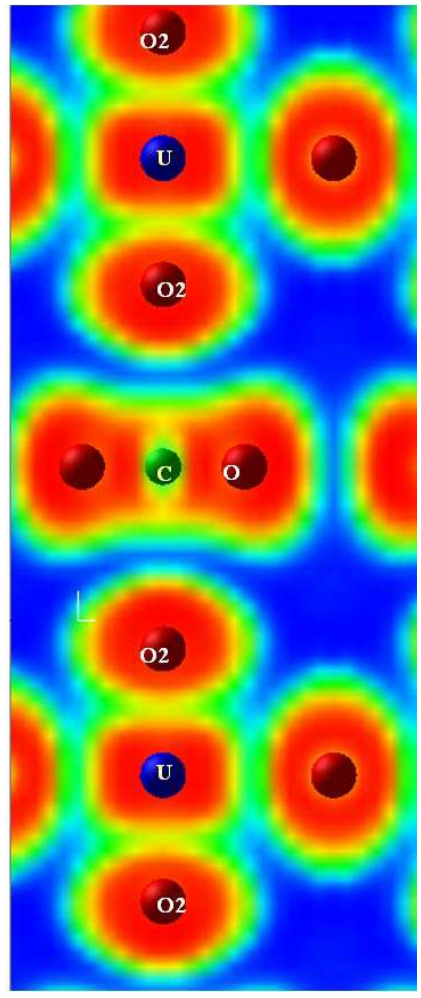

(a)

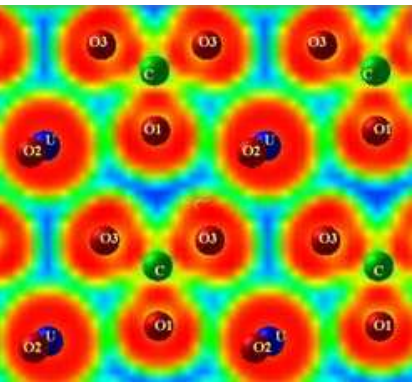

(b)

Figure 2. Electron localization function ELF maps in $\mathrm{UO}_{2} \mathrm{CO}_{3}$ : a) vertical plane along b-axis containing O2-U-O2 linear uranyl groups and b) horizontal plane with carbonate groups, showing 4 adjecent cells. Atom labels correspond to the text and Table 1. 


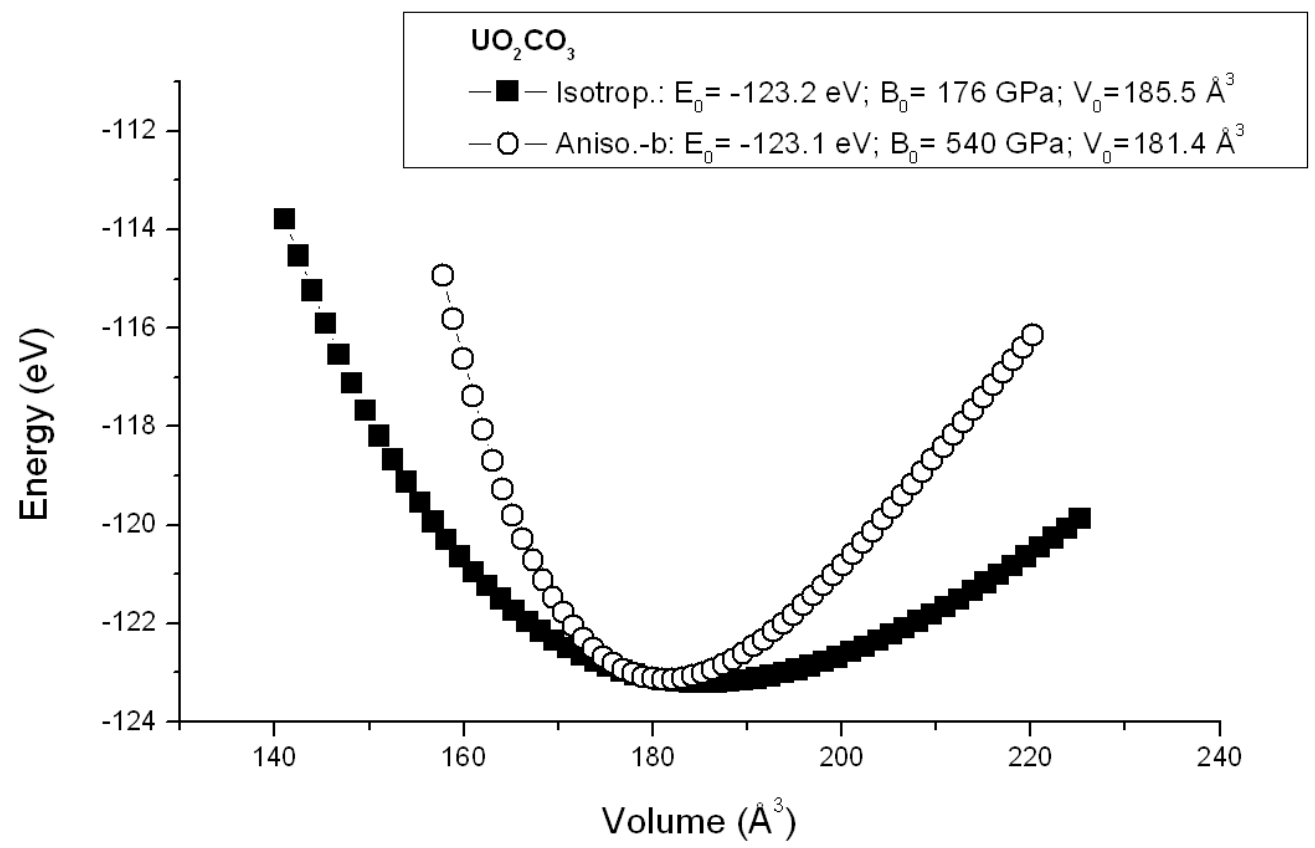

Figure 3. Energy versus volume in $\mathrm{UO}_{2} \mathrm{CO}_{3}$ for isotropic and anisotropic volume changes.
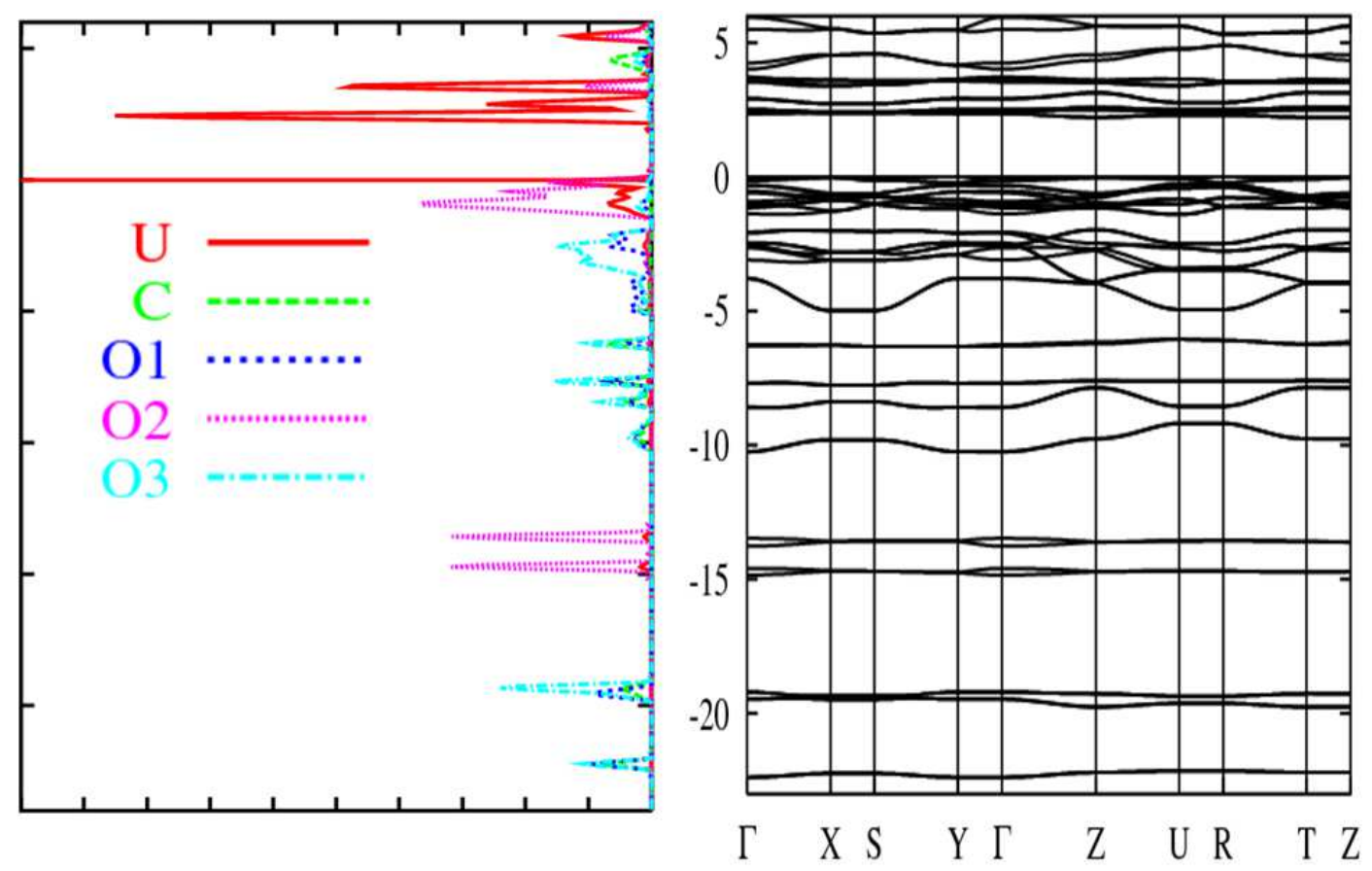

Figure 4. (color online) $\mathrm{UO}_{2} \mathrm{CO}_{3}$ : Electronic band structure and corresponding PDOS. 


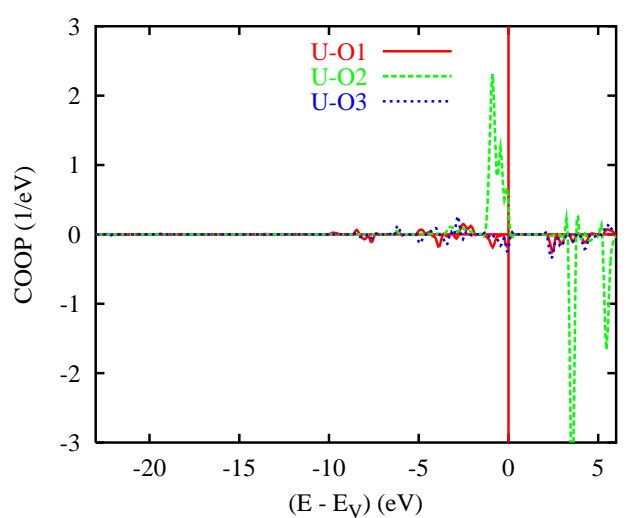

(a)

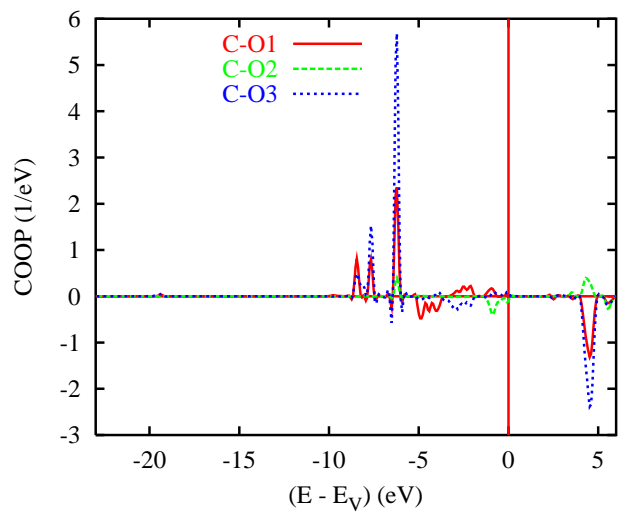

(c)

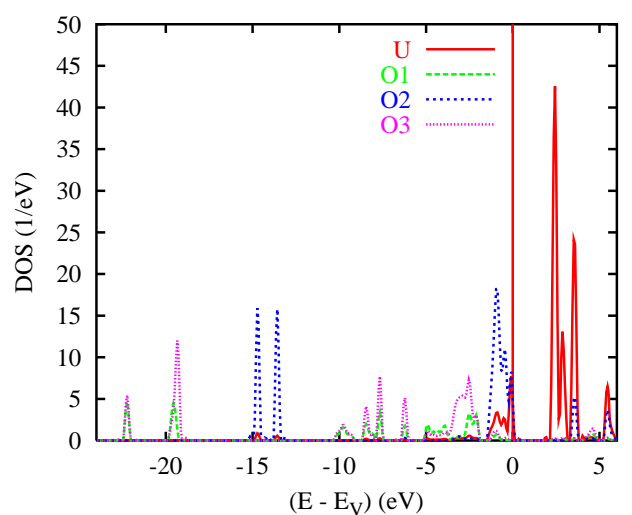

(b)

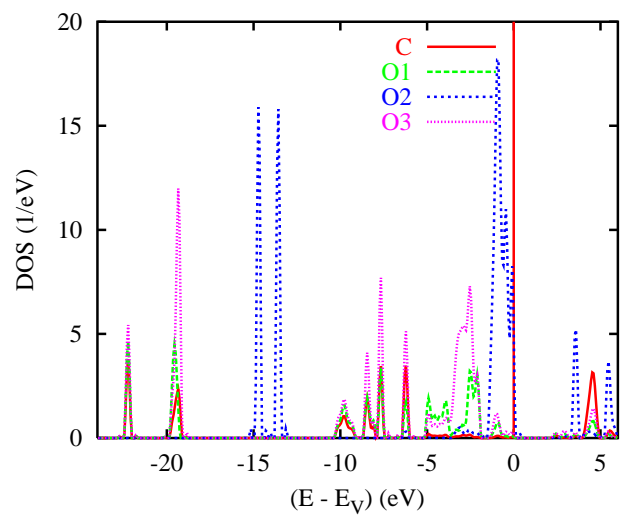

(d)

Figure 5. Chemical bonding and corresponding partial DOS within $\mathrm{UO}_{2} \mathrm{CO}_{3}$ : a) U-O bonding, b) U, O PDOS; c) C-O bonding and d) C, O PDOS. 